\title{
Acanthamoeba lugdunensis
}

National Cancer Institute

\section{Source}

National Cancer Institute. Acanthamoeba lugdunensis. NCI Thesaurus. Code C119302.

A species of motile, unicellular eukaryotic amoebae within the family of

Acanthamoebidae. A. lugdunensis is placed in morphological group II with a 18s rRNA gene sequence type of T4. Members of this group are characterized by the presence of wrinkled ectocysts and endocysts which could be stellate, polygonal, triangular, or oval. This species is a human pathogen and may cause granulomatous amoebic encephalitis and amoebic keratitis. 